\title{
A NOTE ON THE SUPPORT OF RIGHT INVARIANT MEASURES
}

\author{
N.A. TSERPES \\ Department of Mathematics \\ University of Patra \\ Patra, Greece \\ (Received June 6, 1990)
}

ABSTRACT. A regular measure $\mu$ on a locally compact topological semigroup is called right invariant if $\mu(K x)=\mu(K)$ for every compact $K$ and $x$ in its support. It is shown that this condition implies a property reminiscent of the right cancellation law. This is used to generalize a theorem of A. Mukherfea and the author (with a new proof) to the effect that the support of an $r^{*-1}$-invariant measure is a left group iff the measure is right invariant on its support.

KEY WORDS AND PHRASES. Topological semigroup, left group, right invariant (Borel) measure, r*-invariant measure, support of a Borel measure, locally compact semigroup. 1980 AMS SUBJECT CLASSIFICATION CODE. 22A15, 22A20, 43A05, $28 C 10$.

1. INTRODUCTION.

In what follows $S$ will denote a $T_{2}$ locally compact topological semigroup (jointly continuous multiplication) and $\mu$ a positive regular (Radon) measure on the Borel $\sigma$-algebra of $S$ with support $F=\{s \in S$; for every open $V \supset s, \mu(V)>0\}, a s$ in $[1]$ and [2]. We shall use the notation $B x^{-1}=t_{x}^{-1}(B)=\{s \varepsilon s ; s x \varepsilon B\}, t_{x}$

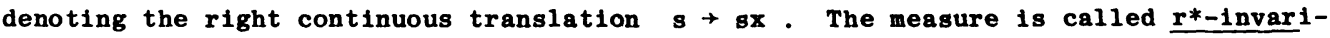
ant on $S$ if $\mu\left(\mathrm{Bx}^{-1}\right)=\mu(B)$ for all Borel $B$ and $x$ in $S$. Such measures received considerable attention in the past in connection with the (still unsolved) conjecture of L.N. Argabright (Proc. Amer. Math. Soc. 17 (1966), 377-382) that their support is a left group 1.e., $F$ is left simple (Fx $=F$ for all $x$ in $F$ ) and right cancellative (equivalently, if it $1 \mathrm{~s}$ left simple and contains an idempotent element). The measure $\mu$ is called right invariant on its support if

$$
\mu(\mathrm{Kx})=\mu(\mathrm{K}) \text { for every compact } \mathrm{K} C F \text { and every } x \in F
$$

In [1] A. Mukherjea and the author proved the "rather tight" result

THEOREM 1. The support of an $r^{*}$-invariant measure on $S$ is a left group iff the measure is right invariant on its support.

Professor Mukherjea in a meeting at University of South Florida asked the questions (1) whether the "intriguing" condition (1.1) (Introduced by himself) implies some sort of right cancellation on $F$ in view of the fact proven by Rigelhof [3] that (1.1) plus that the $t_{x}^{\prime} s, x \in F$, are open maps, imply right cancellation on $F$. (11) Whether Theorem 1 (above) can be substantially generalized. In this note we show: As for question (i) indeed there is a generalized" right cancellation on $S$ (See 
Lemma 1 , below) but as for question (1i), Theorem 1 cannot substantially be generalizcd except that we may only assume that $\mu\left(B x^{-1}\right) \geq \mu(B)$ for every Borel $B$ and every $x \in F$. (Unlihe condition (1.1), no extra generality is obtained whether we assume $B(S$ or $B(F)$. Moreover, our proof (although patented on that of [1]) does not use the functional analytic apparatus of [1] since it uses a version of cancellation from the intrinsic properties of the measure.

2.

We begin by showing in what sense $S$ is pre-right cancellable mod $F$.

LEMMA 1. Let $\mu$ be right invariant on 1 ts support (1.e., $\mu$ satisfies (1.1)). Then

(i) If for $f_{1}, f_{2}, f_{3} \in F, f_{1} f_{2}=f_{3} f_{2}$, then $f_{1}=f_{3}$ for every $f \in F$ that is, we can cancel on the right by premultiplying by any element of the support.

(1i) If $F$ is also a right ideal of $s$, then for $s_{1}, s_{3} \varepsilon s, f_{2} \varepsilon F$, the equation $s_{1} f_{2}=s_{3} f_{2}$ implies $f_{1}=f_{3}$ for all $f \in \overline{F F}=\operatorname{closure}(F F)$ and in particular for any idempotent element $e \in F$.

PROOr. We shall argue by contradiction as in Rigelhof [3,p. 175]. We prove (ii): (The proof of (1) is duite similarly). Assume $s_{1} \mathfrak{l}_{2}=s_{3} \mathfrak{f}_{2}$ but $\mathrm{fs}_{1} \neq \mathrm{fs}_{3}$ so that we can find disjoint compact neighborhoods $U$ and $V$ respectively of these two distinct points (with $f$ some point in $\overline{F F}$ ). Now $\mathrm{Us}_{1}^{-1} \cap \mathrm{Vs}_{3}^{-1}$ must contain a compact neighborhood $W$ of $P$ which in turn must contain a right translate of some compact neighborhood of the form $K \varphi$ for some $\varphi \in F(1 \varepsilon \overline{F F}), 1 . e$. ,

$$
\begin{aligned}
& \mathrm{k} \varphi \subset v \subset \mathrm{Us}_{1}^{-1} \cap \mathrm{vs}_{3}^{-1} \text {, so that } \\
& \left.\left.\mu(K)+\mu(K)=\mu\left(K \varphi \mathbf{s}_{1}\right)+\mu\left(K \varphi \mathbf{s}_{3}\right)=\mu\left(K \varphi \mathbf{s}_{1} \cup \mathbf{K} \varphi \mathbf{s}_{3}\right) \mathbb{1}_{2}\right)=\mu(K \cup \mathbf{K}) \varphi \mathbf{s}_{1} \mathbf{f}_{2}\right)=\mu(\mathrm{K}),
\end{aligned}
$$

which is a contradiction.

COROLLARY 1. Let $\mu$ satisfy (1.1). Then For any pair of idempotents $e_{1}, e_{2} \varepsilon F$, we have $e_{1} e_{2}=e_{1}$ so that the idempoter ts in $F$ form a left-zero subsemigroup of $F$.

(iii) If $\angle y z y x=z y x$ for $x, y, z \in F$, then $z y$ is 1dempotent.

'ROOF. (1): It Hollows since $e_{1} e_{2}=e_{1} e_{2} e_{2}$ and by $L \in m a n$ we way caucel $e_{2}$ by premultiplying by $e_{1}$ and use the fact that $e_{1}$ is idempotent. (1i):Similarly by the above Lemma. (iji): First cancel $x$ by premultiplying by $y$ and then cancel zy by premultiplying by $z$ and obtain zyzy $=z y$.

NC' we are ready to give the generalization of Theorem 1 as follows:

THEOREM 2. Suppose $\mu$ satisfies

$$
\mu\left(B f^{-1}\right) \geq \mu(B) \text { for every Borel } B \text { and every } f \in F
$$

Then $F$ is a left group iff $\mu$ satisfies (1.1).

PROOF. Clearly (1.1) plus inner regularity of $\mu$ imply $\mu\left(B f^{-1}\right) \leq \mu(B)$ for all Borel $B$ and $f \in F$ so that we have $\left(B f^{-1}\right)=\mu(B)$ for every $f \in F$ and Borel $B$. Also (2.1) implies that $\overline{F f}=F$ for all $f \in F$. In the proof of Theorem 1 in [1], 
we produced an idempotent $e$ in Fa, for a $\varepsilon F$, so that $F e=\overline{F e}=F C F a$ and so $\mathrm{Fa}=\mathrm{F}$ for all a $\varepsilon F$ (cf. [1],p. 974). Now, the same proof goes through without any difficulty except that instead of the right cacellation on $F a, a \varepsilon F$, we use Corollary 1 (iii) above.

We give next a result summarizing certain important conditions on $F$ and $\mu$ that are equivalent to $F$ being a left group.

COROLLARY 2. For a locally compact second countable semigroup $S$ admitting an $r^{*-1}$ variant measure $\mu$, these are equivalent:

$$
F \text { is right cancellable }
$$

(ii) $\mu$ is right invariant on its support, 1.e., satisfies (1.1))

(iii) S is pre- right cancellative with respect to $F, 1 . e ., s_{1} s_{2}=s_{3} s_{2}$ with $s_{1}, s_{2}, s_{3} \in F$, implies $f s_{1}=f s_{3}$ for all $f \in F$.

(iv) $\quad F$ is a left sroup.

(v) $\quad F$ has the right translations $t_{f}$ closed for all $f \in F$.

(vi) $F$ has the right translations open and $\mu$ satisfies (1.1).

REMARK. It is not known to our knowledge if (v) and (iv) are equivalent in the absence of second countability.

PROOF. Most of these follow from Theorem 1 or Theorem 2. Note that right cancellation implies that $t_{f}$ are one-to-one and for compact $K, K x x^{-1} \cap F=k x$, so that right invariance on 1 ts support follows from $r *-$ invariance, so (i) $\Rightarrow(1 i) \Rightarrow$ (iii) $\Rightarrow$ (iv) (cf. Theorem 2). Since $F$ is metrizable being regular the technique in [4] for producing an idempotent in Fx applies and thus $F$ becomes a left group. By the result of Rigelhof, (v1) Implies (1).([3]p.175). For (111), see our Lemma 2, below.

REMARK. The following will show the "tightness" of the conditions of Theorem 2. It is well known that a property that "melds" naturally (at first sight) with condition (1.1) is that of lower $\mathrm{r}^{*-i n v a r i a n c e, ~ 1 . e ., ~ t h a t ~} \mu\left(\mathrm{Bx}^{-1}\right) \leq \mu(B)$ for all Borel $B C S$ and $x$ : $S$, for 1 t and (1.1) are equivalent to the condition (cf. [2] and $[5, \mathrm{p} .92])$

$\mu(K x) \geq \mu(K) \quad$ for all compact $K \subset S$ and $x \in S$ with

this inequality becoming equality whenever $K$ and $x$ are in $F$.

This condition (2.2) implies that $F$ is a right ideal and $F e=F$ for ivery idempotent $e \in S$, but these are not enough to make Theorem 2 valid, for the example of $[0, \infty)$ with addition and Lebesgue measure shows that $\mu$ is not $r^{*}$-invariant (it does not satisfy (2.1) of Theorem 2). However this $S$ is pre- right cancellative as the following Lemma generally indicates.

LEMMA 2. Suppose $\mu$ satisfies (2.2) and suppose that $s_{1} s_{2}=s_{3} s_{2}$ for $s_{1}, s_{2}, s_{3} \varepsilon$ s. Then $f s_{1}=f s_{3}$ for all $f \varepsilon \overline{F F}$. If moreover $s_{1} s_{2} \varepsilon F$, then fs $s_{1} f s_{3}$ for a11 $f \in F$.

PROOF. Suppose first that $s_{1} s_{2} \notin F$. Then the second equality in the proof of Lc nma 1 (ii) with $f_{2}$ replaced by $s_{2}$ becomes less or equal and the last remains equality and thus a contradiction obtains. Next assume that $s_{1} s_{2}=s_{3} s_{2}$ and $s_{1} s_{2} \varepsilon$ F. Again, as before (See proof of Lemma 1) there are disjoint compact neighborhoods 
$U, V$ of $\mathrm{fs}_{1}, \mathrm{fs}_{3}$ respectively, such that the intersection of $\mathrm{Us}_{1}^{-1}$ and $\mathrm{Vs}_{3}^{-1}$ contain a compact neighborhood $W$ of $f$ (we use $w \cap F$ instead of $W$ ). Then we have again the inequality

$$
\left.\left.\mu(w)+\mu(w) \leq \mu\left(W s_{1}\right)+\mu\left(W s_{3}\right) \leq \mu\left(W s_{1} \cup W s_{3}\right) s_{2}\right)=\mu(W \cup W) s_{1} s_{2}\right)=\mu(W),
$$

again a contradiction.

REMARK. The most difficult part in problems involving the nature of the support $F$ is producing an idempotent element in $F x$ or in $F$ itself. For this, it would be intersting to have a "survey paper" giving all known methods for producing an idempotent in the presence of measure and/or topological invariance conditions. Apart for haviff some conpact subsemigroup or a compact 1 lber $x x^{-1} \neq \emptyset$ or a two-sided version of (2.2) and a subsemigroup of positive finite inner measure, we know only the technique in [1] which is in some : nse an adoptation of a method of Gelbaum and Kalisch (Canad. J. of Math. 4 (1952), 396-4C:), and the technique of [4] which needs metrizability !. (For example, when the $t_{x}$ are closed mappings, can the "ontoness" of the $t_{x}$ be used to prove that the operator $\pi_{s} f(x)=f(x s)$ on $L_{2}(S, \mu)$ is onto in the non-second countable case? (that will suffice to prove that the support of an $r^{*}$-invariant measure is a left group when the $t_{x}$ 's are closed).

\section{REFERENCES}

1. MUKHERJEA, A. and TSERPES, N.A. A problem on $r^{*}$-invariant measures on locally compact semigroups, Indiana Univ. Math. J 21 (1972), 973-977.

2. TSERPES, N.A. and MUKHERJEA, A. On certain conjectures on invariant measures on semigroups, Semigroup Forum 1 (1970), 260-266.

3. RIgelhof, R. Invariant measures on locally compact semigroups, Proc. Amer. Math. Soc. 28 (1971), 173-176.

4. TSERPES, N.A. and MUKHERJEA, A. Mesures de probabilite r $^{*-1}$. semigroup metrique, C.R. Acad. Sc. Paris Ser. A. 268 (1969), 318-9.

5. BERGLUND, J.F. and HOFMANN, K.h. Compact semitopological semigroups dud weakly almost periodic functions, Springer 1967, Lecture Notes in Math. no 42. 


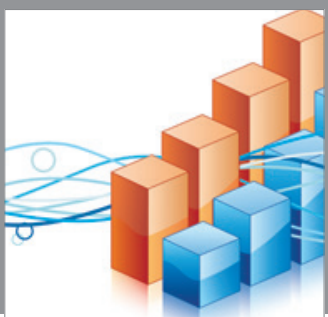

Advances in

Operations Research

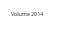

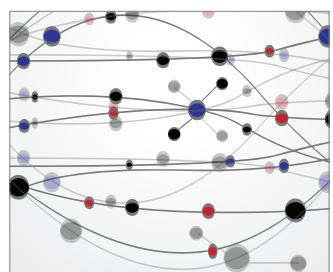

\section{The Scientific} World Journal
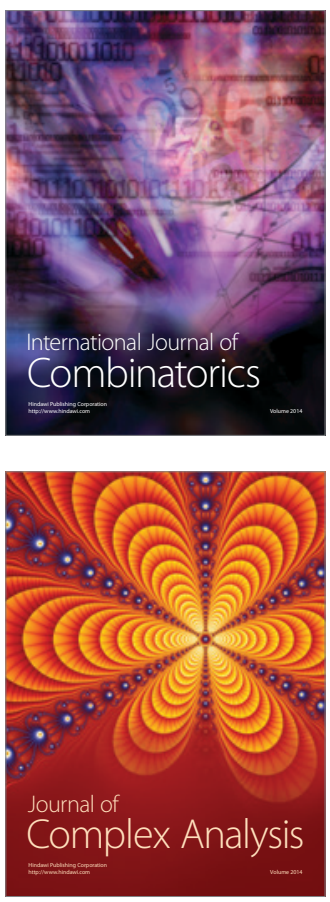

International Journal of

Mathematics and

Mathematical

Sciences
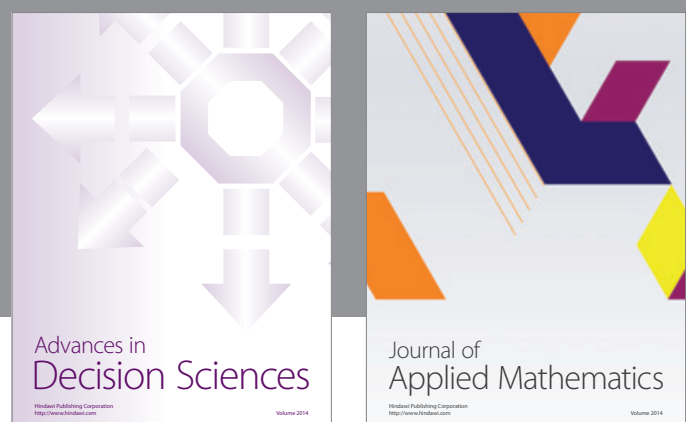

Journal of

Applied Mathematics
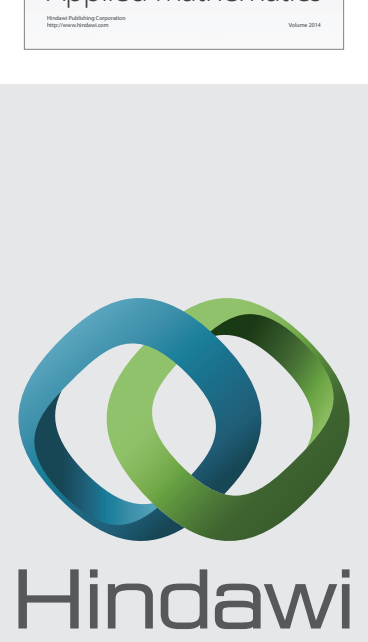

Submit your manuscripts at http://www.hindawi.com
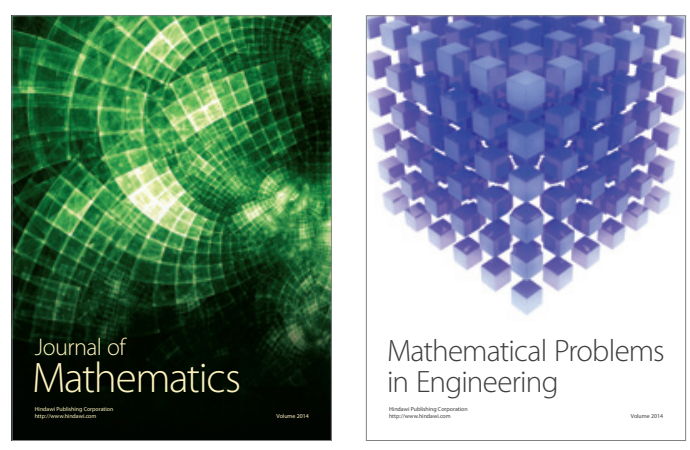

Mathematical Problems in Engineering
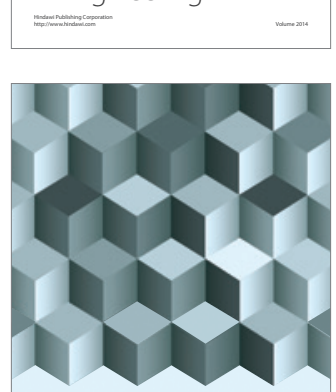

Journal of

Function Spaces
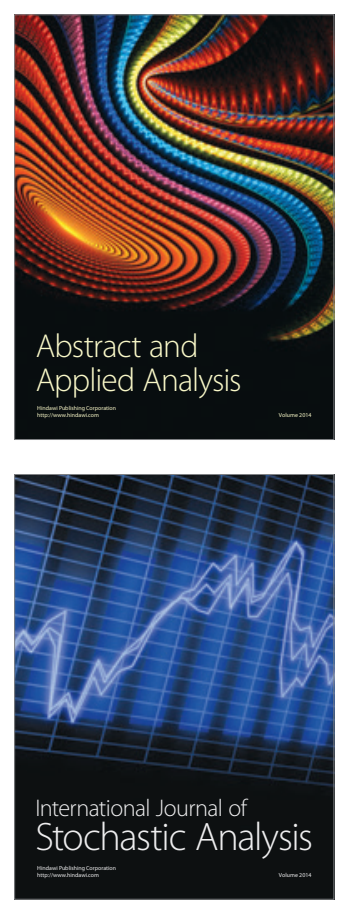

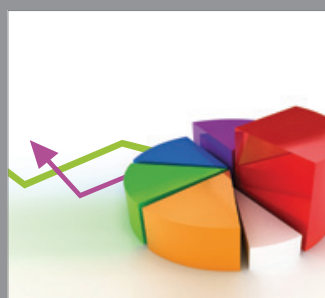

ournal of

Probability and Statistics

Promensencen
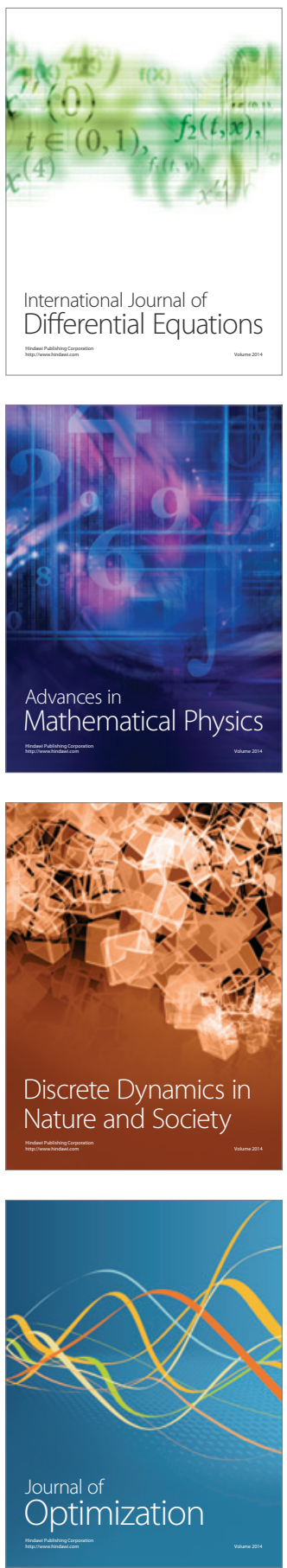